\title{
Association of functional genetic variants of A-kinase anchoring protein 10 with QT interval length in full-term Polish newborns
}

\author{
Beata Łoniewska ${ }^{1}$, Mariusz Kaczmarczyk², Jeremy Simon Clark², Iwona Gorący², \\ Anita Horodnicka-Józwa ${ }^{3}$, Andrzej Ciechanowicz²
}

1Department of Neonatal Disease, Pomeranian Medical University, Szczecin, Poland
2Department of Clinical and Molecular Biochemistry, Pomeranian Medical University,
Szczecin, Poland
3Department of Pediatrics, Endocrinology, Diabetology, Metabolic Diseases
and Children's Cardiology, Pomeranian Medical University, Szczecin, Poland

Submitted: 30 July 2012

Accepted: 13 November 2012

Arch Med Sci 2015; 11, 1: 149-154

DOI: $10.5114 /$ aoms.2013.34172

Copyright @ 2015 Termedia \& Banach

\section{Abstract}

Introduction: A-Kinase Anchoring Proteins (AKAPs) coordinate the specificity of protein kinase A signaling by localizing the kinase to subcellular sites. The $1936 \mathrm{G}$ (V646) AKAP10 allele has been associated in adults with low cholinergic/vagus nerve sensitivity, shortened PR intervals in ECG recording and in newborns with increased blood pressure and higher cholesterol cord blood concentration. The aim of the study was to answer the question of whether 1936A>G AKAP10 polymorphism is associated with the newborn electrocardiographic variables.

Material and methods: Electrocardiograms were recorded from 114 consecutive healthy Polish newborns ( 55 females, 59 males), born after 37 gestational weeks to healthy women with uncomplicated pregnancies. All recordings were made between $3^{\text {rd }}$ and $7^{\text {th }}$ day of life to avoid QT variability. The heart rate per minute and duration of PR, QRS, RR and QT intervals were usually measured. The ECGs were evaluated independently by three observers. At birth, cord blood of neonates was obtained for isolation of genomic DNA.

Results: The distribution of anthropometric and electrocardiographic variables in our cohort approached normality (skewness $<2$ for all variables). No significant differences in anthropometric variables and electrocardiographic traits with respect to AKAP1O genotype were found. Multiple regression analysis with adjustment for gender, gestational age and birth mass revealed that QTc interval in GG AKAP1O homozygotes was significantly longer, but in range, when compared with $A$ alleles carriers (AA+AG, recessive mode of inheritance). No rhythm disturbances were observed.

Conclusions: Results demonstrate possible association between AKAP10 1936A>G variant and QTc interval in Polish newborns.

Key words: A-kinase-anchoring protein 10 , electrocardiograph, genetic polymorphism, newborns, QT interval.

\section{Introduction}

Quantitative non-invasive measures of cardiac electrical activity recorded in electrocardiographic (ECG) studies are widely available in community-based samples and have been found to be predictive of cardiovascular events including sudden cardiac death [1, 2]. Aggregation of these meas-

\author{
Corresponding author: \\ Beata toniewska MD, PhD \\ Department \\ of Neonatal Disease \\ Pomeranian Medical University \\ 72 Powstańców Wielkopolskich St \\ 70-111 Szczecin, Poland \\ Phone: +48914661367 \\ Fax: +48 914661368 \\ E-mail: beatal@sci.pum.edu.pl
}


ures within families suggests a heritable component. The electrocardiographic PR interval has reported heritability estimated at $34 \%$ [3]. The RR interval, or its inverse - heart rate (HR) - has been observed to have a heritability ranging from $32 \%$ to $40 \%$ in family studies [4] and $54 \%$ to $77 \%$ in twin studies [5]. Genome-wide association studies have found, for example, that infants with a QTc $>460 \mathrm{~ms}$ in the first month of life (and with prolonged QT remaining at 1 year) have a $90 \%$ chance of carrying one of a list of mutations from various genes, including KCHN2 and KCNQ1 [6]. The heritability of electrocardiographic QT interval duration, a measure of myocardial repolarization, has been reported to be approximately $35 \%$, indicating that $35 \%$ of the variability in adjusted QT interval duration is attributable to heritable factors [7]. The heritability of ECG suggests there is a significant genetic component to the determination of these measures of myocardial repolarization, sinus node function, atrioventricular conduction, and autonomic function.

The cAMP-dependent protein kinase (protein kinase $A-P K A$ ) is responsible for modulating a vast number of cell physiological processes [8]. A-kinaseanchoring proteins (AKAPs) coordinate PKA signaling by localizing PKA to subcellular sites through binding to the $\mathrm{N}$-terminus of the $\mathrm{R}$ subunits [9]. AKAP10 binds both type I and type II R subunit isoforms of PKA. In a comparison of $~ 6500$ singlenucleotide polymorphisms located in approximately 5000 genes between age-stratified, healthy, European-American individuals, Kammerer et al. found that only the $1936 \mathrm{~A}>\mathrm{G}$ transition in the gene encoding AKAP10 (AKAP10), causing the substitution of isoleucine by valine at position $646(1646 \mathrm{~V}$, $\mathrm{G}$ alleles), was statistically significantly less frequent in older subjects for both men (60 to 79 years) and women (60 to 69 years) [10]. The $1646 \mathrm{~V}$ polymorphism enhances by threefold binding of AKAP10 to PKA Rl $\alpha$ and the targeting of the Rl $\alpha$ subunit to the mitochondria [10]. Analysis of 417 white twin pairs from the adult twin registry at St. Thomas Hospital, London, indicated that the $1646 \mathrm{~V}$ variant was associated with a significant decrease in the electrocardiogram PR interval [10]. Further studies revealed that $1936 \mathrm{~A}>\mathrm{G} A K A P 10$ was associated in adults with increased basal HR and decreased heart rate variability (HRV), which are markers of low cholinergic/vagus nerve sensitivity [11, 12] - one of the factors responsible for increased cardiovascular risk [13].

Therefore, it raises the question whether the 1936A>G AKAP10 transition is associated with electrocardiographic intermediate phenotypes in fullterm Polish newborn infants. Note that it was of importance to measure QTc as early as possible (after the $2^{\text {nd }}$ day of life to avoid maximal QT variability) because a large prospective study has shown that some infants with prolonged QT in the first week are prone to sudden death [14]. In addition to the factors mentioned above, it should be noted that phenotype-genotype correlation studies using newborns seem to be more plausible than those with older individuals due to exclusion of confounding environmental influences such as diet, lifestyle, smoking or disease [15] and the possibility of a very early prophylactic procedure.

\section{Material and methods}

\section{Study subjects}

The study was approved by the local ethics committee and the parents gave informed consent. At the Department of Neonatal Diseases of Pomeranian Medical University in Szczecin, we prospectively recruited 114 consecutive healthy Polish newborns (55 females and 59 males), born after the end of the $37^{\text {th }}$ week of gestation to healthy women with uncomplicated pregnancies in whom ECGs were recorded. Due to artifacts, eleven electrocardiograms had to be excluded. All the recordings were made between the third and seventh day of life in order to avoid the variability of QT interval that is maximal during the first 2 days of life as well [16]. None of the enrolled infants had an episode or history of an acute life-threatening event (including apnea), cardiovascular disease, or disease known to affect the autonomic nervous system. All children were breast-fed and free of medication (i.e. without medicines known to influence heart rate and/or blood pressure). Twins, infants of mothers with preeclampsia, hypertension of any cause, diabetes, autoimmune diseases, history of illicit substance use or antenatal steroid therapy were excluded. Other exclusion criteria were congenital infection, intra-uterine growth restriction (i.e. below the $10^{\text {th }}$ percentile birth mass, length or head circumference), chromosomal aberrations or congenital malformations. At birth, cord blood of neonates was obtained for isolation of genomic DNA. The gender of the newborn, birth mass, body length and head circumference were taken from standard hospital records. Body surface area (BSA) was calculated as the square root of (length $(\mathrm{cm}) \times$ mass (kg)/3600) according to Mosteller [17]. Because only healthy children were included in the study blood electrolyte concentrations were not quantified.

\section{Electrocardiography}

Between days 3 and 7 of life all subjects underwent a routine standard 12-lead body surface electrocardiogram, recorded at a paper speed of $50 \mathrm{~mm} / \mathrm{s}$ and at a gain of $10 \mathrm{~mm} / \mathrm{mV}$ (ACARD 3 apparatus). During the electrocardiographic recording, calm infants were resting in a horizontal recumbent position and breathing freely. If the newborns did not 
become quiet by themselves they were given $5 \%$ glucose to drink to quieten them. The heart rate per minute and duration of PR, QRS, RR and QT intervals were usually measured in lead II from three non-consecutive beats. If records in lead II were unreadable we used lead $\mathrm{V}_{2}$ to measure the above-mentioned parameters (as in Cowan et al. [18], and Rautaharju et al. [19]). The ECGs were evaluated independently by three observers (a child cardiologist and two ECG technicians experienced in child ECG analysis), who were blinded to the infants' identity and to each other's readings. The arithmetic means of the results were compared with results of analysis by a pediatrician, who used a computer program constructed to allow analysis of the scanned electrocardiograms in great detail. There were no significant differences between ECG parameters obtained by all involved investigators. The QTc interval was calculated using the Bazett formula $(\mathrm{QTC}=\mathrm{QT} / \sqrt{ } \mathrm{RR})$. We used Bazett's formula because it is recommended by guidelines for the interpretation of neonatal electrocardiograms of the European Society of Cardiology [20]. This formula is applicable if the heart rate is in the range 80-180 beats/min [21] and the heart rate of newborns observed in our study was in the range 97-174 beats/min.

\section{Determination of AKAP10 genotype}

Genomic DNA from cord blood was isolated (QIAamp Blood DNA Mini Kit; QIAGEN, Hilden, Germany). To genotype the isoleucine to valine mutation at amino acid 646, caused by an A-to-G transition at nucleotide 1936 of the AKAP10 gene, we used our own PCR-RFLP method as described previously. Briefly, genomic DNA was amplified by PCR with primers flanking the polymorphic region: 5'-TCGGTGTTAGGTATCCAGTAGTTG-3' as the sense primer, and 5'-TAAGAAGGTAATCCCCACAGCAGT-3' as the antisense primer. These primers yielded a PCR product of $437 \mathrm{bp}$ in length. Genomic DNA samples (20 ng) were amplified in a final volume of $10 \mu \mathrm{l}$, which contained $2 \mathrm{pM}$ of each primer, $0.25 \mathrm{mM}$ of each deoxyribonucleotide (dATP, dTTP, dCTP, dGTP) (Promega), $50 \mathrm{mM} \mathrm{KCl}, 20 \mathrm{mM}$ Tris- $\mathrm{HCl}$ ( $\mathrm{pH}$ 8.4), $1.5 \mathrm{mM} \mathrm{MgCl}_{2}, 10 \mathrm{mg} / \mathrm{ml}$ albumin from nuclease-free bovine serum, and $0.25 \mathrm{U}$ of DNA polymerase (Taq, Boehringer Mannheim, Penzberg, Germany). The amplification was performed in a gradient thermocycler (Mastercycler, Eppendorf, Warsaw, Poland) using the following conditions: initial denaturation at $94^{\circ} \mathrm{C}$ for $5 \mathrm{~min}$, then 36 cycles: denaturation at $94^{\circ} \mathrm{C}$ for $20 \mathrm{~s}$, annealing at $64^{\circ} \mathrm{C}$ for $40 \mathrm{~s}$, and extension at $72^{\circ} \mathrm{C}$ for $40 \mathrm{~s}$. The reaction finished with a final extension at $72^{\circ} \mathrm{C}$ for $8 \mathrm{~min}$. Subsequently, the products of amplification were incubated at $65^{\circ} \mathrm{C}$ for $3 \mathrm{~h}$ with $1 \mathrm{U}$ of restriction enzyme Tai I (MBI Fermentas, Vilnius, Lithuania), and separated using 3\% agarose gel electrophoresis, and stained with ethidium bromide. The "wildtype" sequence (1936A allele) was identified by lack of the Tai I restriction site, while the $1936 \mathrm{G}$ allele was digested into 269 and 168 bp restriction fragments. Results were recorded with photographs of gels under UV light. All samples were independently genotyped using a blind method in duplicate, i.e. all samples were anonymously labeled by one person and then genotyped by the second person.

\section{Statistical analysis}

The divergence of AKAP1O genotype frequencies from Hardy-Weinberg equilibrium was assessed using a $\chi^{2}$ test. The distribution of each quantitative variable was tested for skewness. Quantitative data were presented as means $\pm \mathrm{SD}$. Association between $A K A P 10$ genotype and each outcome variable was assessed by one-way ANOVA. In addition, electrocardiographic intermediate phenotypes (traits), with respect to dominant and recessive mode of inheritance of the 1936G AKAP10 minor allele, were analyzed by multiple regression with adjustment for confounding variables. Statistical significance was defined as $p<0.05$. All data were analyzed using a data analysis software system (Statistica, version 8.0, StatSoft, Inc. 2007, www. statsoft.com).

\section{Results}

All PCR-RFLP samples were genotyped twice and a concordance rate of $100 \%$ was attained. There were $41 \mathrm{AA} A K A P 10$ homozygotes (36.0\%), 59 AG heterozygotes (51.7\%) and 14 GG AKAP10 homozygotes (12.3\%). The frequency of the $1936 \mathrm{G}$ AKAP10 minor allele was $38.2 \%$ (35.6\% in newborn males and $40.9 \%$ in newborn females). The AKAP10 genotype distribution conformed to the expected HardyWeinberg equilibrium. The distribution of anthropometric (Table I) and electrocardiographic variables in our cohort approached normality (skewness $<2$ for all variables). Mean body mass was $3409 \pm 530 \mathrm{~g}$, body length $56.2 \pm 3.3 \mathrm{~cm}$, head circumference 33.7 $\pm 1.5 \mathrm{~cm}$, body surface area $0.23 \pm 0.02 \mathrm{~m}^{2}$. All infants were born in good condition (Apgar score was 8 or higher). No significant differences in anthropometric variables and electrocardiographic traits with respect to $A K A P 10$ genotype were found. Multiple regression analysis with adjustment for gender, gestational age and birth mass revealed that QTc interval in GG AKAP10 homozygotes was significantly longer when compared with A allele carriers (AA+AG, recessive mode of inheritance) (Table II).

\section{Discussion}

The results of our study show an association between the 1936A>G (I646V) AKAP10 genotype 
Table I. Background characteristics (means \pm SD) of newborns with respect to AKAP10 genotypes

\begin{tabular}{|lcccccc|}
\hline Variables & \multirow{2}{*}{ Total } & \multicolumn{3}{c}{ Genotype } & \multirow{2}{*}{ Value of $p_{\text {ANOVA }}$ Value of $p_{\text {Do }}$} \\
\cline { 3 - 5 } & & AA & AG & GG & & \\
\hline Gestational age [weeks] & $39.9 \pm 1.0$ & $39.7 \pm 0.9$ & $40.1 \pm 1.0$ & $39.8 \pm 0.9$ & 0.110 & 0.085 \\
\hline Birth weight $[\mathrm{g}]$ & $3409 \pm 540$ & $3383 \pm 518$ & $3403 \pm 523$ & $3540 \pm 537$ & 0.613 & 0.927 \\
\hline Body length [cm] & $56.2 \pm 3.3$ & $56.1 \pm 3.4$ & $56.3 \pm 3.1$ & $56.6 \pm 3.3$ & 0.887 & 0.860 \\
\hline Head circumference $[\mathrm{cm}]$ & $33.7 \pm 1.5$ & $33.7 \pm 1.6$ & $33.7 \pm 1.4$ & $33.8 \pm 1.5$ & 0.954 & 0.881 \\
\hline Body surface area $\left[\mathrm{m}^{2}\right]$ & $0.231 \pm 0.023$ & $0.229 \pm 0.024$ & $0.230 \pm 0.023$ & $0.235 \pm 0.024$ & 0.685 & 0.968 \\
\hline
\end{tabular}

Multiple regression: $p_{D O}$ for dominant $(G G+A G$ vs. $A A) ; p_{R E}$ for recessive $(G G$ vs. $A G+A A)$ models of inheritance.

Table II. ECG characteristics (means $\pm \mathrm{SD}$ ) in newborns with respect to AKAP10 genotypes

\begin{tabular}{|c|c|c|c|c|c|c|}
\hline \multirow[t]{2}{*}{ Variables } & \multicolumn{3}{|c|}{ AKAP10 genotype } & \multirow[t]{2}{*}{ Value of $p_{\text {ANOVA }}$} & \multirow[t]{2}{*}{ Value of $p_{\mathrm{DO}}$} & \multirow[t]{2}{*}{ Value of $p_{\mathrm{RE}}$} \\
\hline & AA & AG & GG & & & \\
\hline HR [beats/min] & $142 \pm 21$ & $143 \pm 24$ & $142 \pm 15$ & 0.997 & 0.825 & 0.999 \\
\hline $\mathrm{PR}[\mathrm{ms}]$ & $122 \pm 13$ & $122 \pm 10$ & $122 \pm 6$ & 0.998 & 0.707 & 0.888 \\
\hline $\mathrm{PQ}[\mathrm{ms}]$ & $89 \pm 12$ & $89 \pm 10$ & $91 \pm 9$ & 0.698 & 0.941 & 0.567 \\
\hline QRS [ms] & $74 \pm 4$ & $74 \pm 5$ & $76 \pm 6$ & 0.683 & 0.690 & 0.490 \\
\hline QT [ms] & $258 \pm 22$ & $258 \pm 27$ & $266 \pm 15$ & 0.642 & 0.548 & 0.397 \\
\hline QTc [ms] & $395 \pm 17$ & $393 \pm 20$ & $408 \pm 25$ & 0.077 & 0.562 & 0.030 \\
\hline
\end{tabular}

Multiple regression: $p_{D O}$ for dominant $(G G+A G$ vs. $A A) ; p_{R E}$ for recessive $(G G$ vs. $A G+A A)$ models of inheritance after adjustment for gender, gestational age and birth mass.

and length of the QTc interval in Polish newborns born full term. The observed value of the QTc interval is in accordance with other studies of healthy newborns. In a study performed by Schwartz et al. on 43080 white neonates, the $97.5^{\text {th }}$ and the $50^{\text {th }}$ percentiles, i.e. upper and normal values of QTC, were $443 \mathrm{~ms}$ and $364 \mathrm{~ms}$ respectively [6]. None of the newborns in our study had a QTc interval longer than the upper normal limit of QTc, which is considered to be $440 \mathrm{~ms}$ [14]. Because only $2.5 \%$ of normal newborns can be expected to have greater length of this interval, we probably did not observe this due to the small sample size. We do not think that the values observed in our study are of clinical significance and/or require any special monitoring of newborns. However, they should shed some light on the influence of AKAP1O on the physiology of the developing heart.

This study examined the association of a functional AKAP1O polymorphism with surface ECG investigation and the association of genetic polymorphism with surface ECG in a cohort of newborns within a few days after birth. Previously, Kammerer et al. revealed an association between AKAP10 polymorphism and shorter PR interval in 97 traits using a sample of 417 white twin pairs [10]. They did not observe any changes concerning the QT interval or T wave.

Żukowski et al. reported that during kidney transplantation patients with the 1939A $>$ G AKAP10 variant had a significantly longer QTc interval, which declined to a level similar to that in the control group $1 \mathrm{~h}$ after the operation [22]. These data suggest that this polymorphism can predict arrhythmia in situations with triggering circumstances such as anesthesia and surgical procedures, even in patients who usually have normal values of the QTC interval. There are some differences between our study and the study of Żukowski et al. The QTC interval in our study was measured in healthy newborns, and all conditions which could affect the QTC interval were excluded. Newborns were investigated during rest/sleep and in the first days of life, i.e. during a phase of heart immaturity. The study of Żukowski et al. was performed on chronically sick adult patients, who qualified for renal transplantation and had undergone anesthesia and surgery.

Although during the first days of life newborns are also stimulated by a rapidly changing environment, this situation cannot be compared to renal transplantation. Nevertheless, we could consider the hypothesis that the 1939A $>\mathrm{G} A K A P 10$ polymorphism, with increased signaling of PKA, could possibly increase the risk of cardiac arrhythmia under aggravating conditions. AKAP10 is thought to affect heart rhythm [23]. Tingley et al. generated AKAP10mutant mice, with a mutation which has a lower affinity to PKA [11]. AKAP10-mutant mice showed many cardiovascular abnormalities including decreased heart rate, pathological sinoatrial and atrioventricular conduction as well as arrhythmias and a higher susceptibility to sudden death. It was 
postulated that the observed effects were associated with higher activity of the parasympathetic system in the mutant mice. This could mean that the $1646 \mathrm{~V}$ AKAP10 variant, responsible for enhanced binding to PKA, could have the effect of actually decreasing the activity of the parasympathetic system, associated with cardiovascular risk. It is interesting that $A K A P 10$ mutation caused death only in adult mice.

Similar observations confirming increased parasympathetic activity associated with disruption of AKAP10 binding to PKA were reported by Patel et al. [24]. In experiments performed on isolated cardiomyocytes and on isolated heart it was observed that such disruption reduced myocyte shortening and rates of contraction and relaxation. In the isolated hearts the authors observed a decreased heart rate and contractile function. Because the hearts were isolated from neurohormonal activation, the results suggest that the influence of AKAP10 does not only depend on vagal nerve activation. These experimental observations were confirmed in human studies, which showed that the V646 variant was associated with low cholinergic/vagus nerve sensitivity expressed as increased basal heart rate and decreased heart rate variability [11, 12]. In our study we did not observe arrhythmia and we have not found a significant association between $1939 \mathrm{~A}>\mathrm{G}$ AKAP10 polymorphism and heart rate in the newborns studied by us. Our observations are in accordance with $\dot{Z} u$ kowski et al. [22], who also did not observe arrhythmia, or changes of HR and HRV, in their transplant patients. However, when comparing the results obtained by us and the results from the work of Tingley et al. [11] and Neumann et al. [12] extreme caution should be taken because of significant differences in the design of the studies. Our study included newborns in whom heart rate was calculated on the basis of standard surface ECG performed during resting/sleeping. Tingley et al. used $24 \mathrm{~h}$ continuous ECG recording and Neumann et al. used 10 min continuous 2-lead electrocardiograms. Both methods allowed the measurement of HR as well as HRV. Moreover, Tingley et al. did not present HR results for the genotypes of AKAP10, and only showed that carriers of the 1936G (V646) variant $A K A P 10$ were more likely to have a mean $\mathrm{HR}$ (over $24 \mathrm{~h}$ ) of $>75$ beats/min, which is an independent risk factor for sudden cardiac death in the adult population. For our newborns all HR values were $>75$ beats/min. Additionally, in the study performed by Neumann the influence of the AKAP1O polymorphism on HR and HRV was not confirmed in the case of high frequency (HF) HRV. The authors postulated that this AKAP10 variant may have more influence on basal HR and sympathetic-vagal interactions at the sinus node. In newborns the auto- nomic system of the heart is not well balanced due to its immaturity. We also have to note that the influence of the sympathetic and parasympathetic system on the QTC interval is not fully elucidated and data are very often conflicting [25]. Due to these circumstances, the results observed by us concerning QTc interval and HR suggest that further studies are needed to propose any deeper conclusion.

In contrast to Kammerer et al., we did not observe an influence of $A K A P 10$ polymorphism on $P R$ interval in newborns. (The values observed in our study are in the general range found in other literature [14].) The PR interval reflects atrioventricular nodal conduction and can lead to atrial fibrillation. Conduction disturbances associated with AKAP10 were observed in an experimental study performed by Tingley et al. [11], but no human study has confirmed these observations. Taking into consideration our other data, we cannot expect that the observations of Kammerer et al. of an adult population could be confirmed in our newborns, as the $H R$ is too fast to observe PR interval shortening using a standard surface ECG. The underlying molecular mechanism for the relationship between the AKAP10 polymorphism and neonatal surface ECG is unclear. Recently, Eggers et al. reported that the RGS domains of AKAP10 interact with the small GTPases Rab4 and Rab11, which regulate endocytic recycling. Therefore, these authors suggested that the fact that 1939A $>\mathrm{G} A K A P 10$ is associated with disease phenotypes could be the result of altered surface expression of various receptors or channels [26], including adrenergic receptors involved in heart rhythm regulation [27]. It should also be taken into consideration that the PKA-AKAP pathway is involved in water and electrolyte homeostasis, e.g. via modulation of epithelial sodium channel (ENaC) or aquaporin 2 [28].

In conclusion, our results demonstrate a possible association between the 1936A>G AKAP10 variant and QTc in the aboriginal-European newborn population. However, the results should be interpreted with caution due to the relatively small sample size. The results observed by us have no clinical consequences for newborns and do not require any prophylactic treatments, but they do suggest some influence of $A K A P 10$ on the physiology of the developing heart.

\section{Conflict of interest}

The authors declare no conflict of interest.

\section{References}

1. Kannel WB, McGee DL, Schatzkin A. An epidemiological perspective of sudden death. 26-year follow-up in the Framingham Study. Drugs 1984; 28: 1-16. 
2. Straus SM, Kors JA, De Bruin ML, et al. Prolonged QTC interval and risk of sudden cardiac death in a population of older adults. J Am Coll Cardiol 2006; 47: 362-7.

3. Havlik RJ, Garrison RJ, Fabsitz R, Feinleib M. Variability of heart rate, P-R, QRS and Q-T durations in twins. J Electrocardiol 1980; 13: 45-8.

4. An P, Rice T, Gagnon J, et al. Familial aggregation of resting blood pressure and heart rate in a sedentary population: the HERITAGE Family Study. Health, risk factors, exercise training, and genetics. Am J Hypertens 1999; 12: 264-70.

5. Russell MW, Law I, Sholinsky P, Fabsitz RR. Heritability of ECG measurements in adult male twins. J Electrocardio 1998; 30: 64-8.

6. Schwartz PJ, Stramba-Badiale M, Crotti L, et al. Prevalence of the congenital long-QT syndrome. Circulation 2009; 120: 1761-7.

7. Newton-Cheh C, Larson MG, Corey DC, et al. QT interval is a heritable quantitative trait with evidence of linkage to chromosome 3 in a genome-wide linkage analysis: the Framingham Heart Study. Heart Rhythm 2005; 2: 277-84.

8. Amieux PS, McKnight GS. The essential role of RI alpha in the maintenance of regulated PKA activity. Ann N Y Acad Sci 2002; 968: 75-95.

9. Edwards AS, Scott JD. A-kinase anchoring proteins: protein kinase A and beyond. Curr Opin Cell Biol 2000; 12: 217-21.

10. Kammerer S, Burns-Hamuro LL, Ma Y, et al. Amino acid variant in the kinase binding domain of dual-specific A kinase-anchoring protein 2: a disease susceptibility polymorphism. Proc Natl Acad Sci U S A 2003; 100: 4066-71.

11. Tingley WG, Pawlikowska L, Zaroff JG, et al. Gene-trapped mouse embryonic stem cell-derived cardiac myocytes and human genetics implicate AKAP10 in heart rhythm regulation. Proc Natl Acad Sci U S A 2007; 104: 8461-6.

12. Neumann SA, Tingley WG, Conklin BR, et al. AKAP10 (I646V) functional polymorphism predicts heart rate and heart rate variability in apparently healthy, middle-aged European-Americans. Psychophysiology 2009; 46: 466-72.

13. Jouven X, Empana J, Schwartz PJ, Desnos M, Courbon D, Ducimetiere $P$. Heart-rate profile during exercise as a predictor of sudden death. N Engl J Med 2005; 352: 1951-8.

14. Schwartz PJ, Garson A Jr, Paul T, Stramba-Badiale M, Vetter VL, Wren C. Guidelines for the interpretation of the neonatal electrocardiogram. Eur Heart J 2002; 23 : 1329-44.

15. Han T, Wang X, Cui Y, Ye H, Tong X, Piao M. Relationship between angiotensin-converting enzyme gene insertion or deletion polymorphism and insulin sensitivity in healthy newborns. Pediatrics 2007; 119: 1089-94.

16. Schwartz PJ, Salice P. Cardiac arrhythmias in infancy: prevalence, significance and need for treatment Eur Heart J 1984; 5: 43-50.

17. Mosteller RD. Simplified calculation of body-surface area. N Engl J Med 1987; 317: 1098.

18. Cowan JC, Yusoff K, Moore M, et al. Importance of lead selection in QT interval measurement. Am J Cardiol 1988; 61: 83-7.

19. Rautaharju PM, Surawicz B, Gettes LS. AHA/ACCF/HRS recommendations for the standardization and interpretation of the electrocardiogram: part IV: the ST segment, $T$ and $U$ waves, and the QT interval: a scientific statement from the American Heart Association Electrocardiography and Arrhythmias Committee, Council on Clinical Cardiology; the American College of Cardiology
Foundation; and the Heart Rhythm Society. J Am Coll Cardiol 2009; 53: 982-91.

20. Schwartz PJ, Garson A Jr, Paul T, et al. Guidelines for the interpretation of the neonatal electrocardiogram. A task force of the European Society of Cardiology. Eur Heart J 2002; 23: 1329-344.

21. Schwartz PJ, Stramba-Badiale M. Repolarization abnormalities in the newborn. J Cardiovasc Pharmacol 2010; 55: 539-43.

22. Żukowski M, Bohatyrewicz R, Biernawska J, et al. Association of the A1936G (rs203462) of A-kinase anchoring protein 10 polymorphisms with QT interval prolongation during kidney transplantation. Transplant Proc 2009; 41: 3036-8.

23. Diviani D, Dodge-Kafka KL, Li J, Kapiloff MS. A-kinase anchoring proteins: scaffolding proteins in the heart. Am J Physiol Heart Circ Physiol 2011; 301: 1742-53.

24. Patel HH, Hamuro LL, Jo Chun B, et al. Disruption of protein kinase A localization using trans-activator of transcription (TAT)- conjugated A-kinase-anchoring peptide reduces cardiac function. J Biol Chem 2010; 36: 27632-40.

25. Murakawa Y, Yamashita T, Ajiki K, et al. Is the QT interval an indicator of autonomic state? Jpn Heart J 2000; 4: 713-21.

26. Eggers CT, Schafer JC, Goldenring JR, Taylor SS. D-AKAP2 Interacts with Rab4 and Rab11 through its RGS domains and regulates transferrin receptor recycling. J Biol Chem 2009; 284: 32869-80.

27. Cao TT, Deacon HW, Reczek D, Bretscher A, von Zastrow M. A kinase-regulated PDZ-domain interaction controls endocytic sorting of the beta2-adrenergic receptor. Nature 1999; 401: 286-90.

28. Snyder PM, Olson DR, Kabra R, Zhou R, Steines JC. CAMP and serum and glucocorticoid-inducible kinase (SGK) regulate the epithelial $\mathrm{Na}(+)$ channel through convergent phosphorylation of Nedd4-2. J Biol Chem 2004; 279: 45753-8. 